This is an electronic copy of a paper that originally appeared as: Zhu, F., Baker, D., Skommer, J., Sewell, M., \& Wlodkowic, D. (2015). Lab-on-a-chip technology for a non-invasive and real-time visualization of metabolic activities in larval vertebrates. In S. van den Driesche (ed.), SPIE Proceedings 9518: Bio-MEMS and Medical Microdevices II, (pp. 951813-1-951813-6).

Bellingham, WA: SPIE. DOI: 10.1117/12.2180690

Copyright 2015 Society of Photo Optical Instrumentation Engineers. One print or electronic copy may be made for personal use only. Systematic electronic or print reproduction and distribution, duplication of any material in this paper for a fee or for commercial purposes, or modification of the content of the paper are prohibited.

http://dx.doi.org/10.1117/12.2180690 


\title{
Lab-on-a-chip technology for a non-invasive and real-time visualisation of metabolic activities in larval vertebrates
}

\author{
Feng Zhu ${ }^{1}$, Daniel Baker ${ }^{2}$, Joanna Skommer ${ }^{1}$, Mary Sewell ${ }^{3}$, and Donald Wlodkowic ${ }^{1,4,5, *}$ \\ ${ }^{1}$ School of Applied Sciences, RMIT University, Melbourne, VIC, Australia \\ ${ }^{2}$ International Centre for Sturgeon Studies, Vancouver Island University, Nanaimo, BC, Canada \\ ${ }^{3}$ School of Biological Sciences, The University of Auckland, Auckland, New Zealand \\ ${ }^{4}$ Centre for Environmental Sustainability and Remediation (EnSuRe), RMIT University, Melbourne, \\ Australia \\ ${ }^{5}$ Centre for Additive Manufacturing, RMIT University, Melbourne, Australia
}

\begin{abstract}
Non-invasive and real-time visualisation of metabolic activities in living small organisms such as zebrafish embryo and larvae has not yet been attempted due to profound analytical limitations of existing technologies. Significant progress in the development of physico-optical oxygen sensors using luminescence quenching by molecular oxygen has recently been made. Sensing using such microsensors is, however, still performed in small glass chambers that hold single specimens and thus not amenable for high-throughput data acquisition.

In this work, we present a proof-of-concept approach by using microfluidic Lab-on-a-Chip (LOC) technologies combined with sophisticated optoelectronic sensors. The LOC device is capable of immobilising live zebrafish embryos with continuous flow perfusion, while the sensor uses innovative Fluorescence Ratiometric Imaging (FRIM) technology that can kinetically quantify the temporal patterns of aqueous oxygen gradients at a very fine scale based on signals coming from an optical sensor referred to as a sensor foil. By embedding the sensor foil onto the microfluidic living embryo array system, we demonstrated in situ FRIM on developing zebrafish embryos. Future integration of microfluidic chip-based technologies with FRIM technology represents a noteworthy direction to miniaturise and revolutionise research on metabolism and physiology in vivo.
\end{abstract}

Keywords: Microfluidics, Lab-on-a-Chip, microarrays, zebrafish, embryo, metabolic activity, oxygen, sensor foils, fluorescence ratiometric imaging

*e-mail donald.wlodkowic@rmit.edu.au; phone 613992 57157; fax 613992 57110; http://www.rmit.edu.au/staff/donald-wlodkowic

\section{INTRODUCTION}

Significant progress in the development of physico-optical oxygen sensors using luminescence quenching by molecular oxygen has recently been made ${ }^{1,2}$. Sensing using such microsensors is, however, still performed in small glass chambers that hold single specimens and thus not amenable for high-throughput data acquisition ${ }^{3}$. Moreover, conventional measurements can only be taken along one dimension and provide classical, averaged results of metabolic activities of an entire specimen ${ }^{3}$. As such they do not provide any spatial-temporal information of, for example, 2D and 2D+time oxygen distributions inside the living tissues or medium surrounding the specimen ${ }^{3}$. These profound analytical limitations restrict fundamental studies on how abiotic and pharmacological stimuli affect embryonic physiology, the developmental stage when animals are typically considered to be most sensitive to environmental perturbations.

Recently a new generation of Fluorescence Ratiometric Imaging (FRIM) systems based on kinetic quantification of the fluorescence signal coming from an optical sensor often referred to as "sensor foils" have been developed ${ }^{4-6}$. These miniaturised systems can be used to observe the temporal patterns of aqueous oxygen gradients at a very fine scale. In this work, we demonstrate a proof-of-concept microfluidic technology combined with the latest generation of sensor foil

Bio-MEMS and Medical Microdevices II, edited by Sander van den Driesche, Proc. of SPIE Vol. 9518, $951813 \cdot$ • C 2015 SPIE $\cdot$ CCC code: $1605-7422 / 15 / \$ 18 \cdot$ doi: $10.1117 / 12.2180690$ 
oxygen detection system (VisiSens, PreSens Inc., Germany) ${ }^{4-6}$. As an investigative tool, LOCs represent a new direction that may miniaturise and revolutionise research on metabolism and physiology in vivo ${ }^{7-9}$.

In this study, we demonstrate a proof-of-concept microfluidic technology combined with the latest generation of sensor foil oxygen detection system. Lab-on-a-Chip represents a new direction that may miniaturize and revolutionize research on metabolism and physiology in vivo. Our preliminary objective was to determine whether the sensor foil oxygen detection system can be effectively integrated into the chip-based platform and allow for characterization of oxygen gradients with the accuracy and precision needed to estimate embryonic oxygen consumption rates in zebrafish embryos. Our work showcases a new research avenue that enables non-invasive measurement of metabolic activities in larval vertebrates and performed on miniaturized chip-based laboratories.

\section{MATERIALS AND METHODS}

\subsection{Zebrafish husbandry and embryo culture}

Adult wild type zebrafish were held in a recirculating aquatic system on a 14h:10h light:dark cycle. Fish were fed twice daily, once with live crustaceans Artemia sp. and once with dry food. Environmental temperature was maintained at $27.5^{\circ} \mathrm{C}$ and environmental $\mathrm{pH}$ at 7.5-8.0. Zebrafish embryos were obtained from pair-wise mating and natural spawning of the adult zebrafish ${ }^{8,10}$. Eggs were retrieved from spawning groups of four males and three females. Fertilized eggs were collected within an hour of spawning and transferred to embryonic medium (i.e., E3 consisting of $146 \mathrm{mg} / 1 \mathrm{NaCl}$; $6.3 \mathrm{mg} / 1 \mathrm{KCl} ; 24.3 \mathrm{mg} / \mathrm{l} \mathrm{CaCl}_{2} ; 40.7 \mathrm{mg} / 1 \mathrm{MgSO}_{4}$ ). Embryos were cultured in this solution and held in a dark incubator at $28^{\circ} \mathrm{C}$ until use. Animals were treated according to University of Auckland Animal Care regulations.

\subsection{Microfluidic chip fabrication}

A microfluidic system consisted of a 3D multilayer chip-based device fabricated in a biologically compatible and optically transparent PMMA polymer using infrared laser micromachining (Figure 1A) as described earlier ${ }^{8,10}$. The 3D chip comprised of five integrated modules: (i) a main loading channel $(52 \mathrm{~mm} \times 1.8 \mathrm{~mm} \times 1.0 \mathrm{~mm})$ for embryo loading, toxicant delivery and post-analysis embryo recovery; (ii) a linear array of 18 miniaturized embryo traps $(\phi 1.5 \mathrm{~mm} \times 1$ $\mathrm{mm}$ ) beneath the loading channel, for single embryo trapping and immobilization (Figure 1 and B). The dimensions of the wells were designed not only to hold one embryo, but also to ensure that the following embryos would continue through the chip until the next well was reached (Figure 1C). In this way, multiple wells could be rapidly loaded with embryos (Figure 1C). Leaving some microwells unoccupied allowed for an internal control to characterize oxygen levels in the solution over the course of the experiment; (iii) a suction manifold included both interconnecting channels $(0.3$ $\mathrm{mm} \times 1.6 \mathrm{~mm} \times 0.5 \mathrm{~mm})$ for each well and a main suction channel $(53 \mathrm{~mm} \times 2.5 \mathrm{~mm} \times 0.5 \mathrm{~mm})$. The small channels created a suction force to help embryo docking, immobilization and solution delivery (Figure 1A and B). The suction manifold was positioned at the side of traps, providing both upright and inverted imaging capabilities; (iv) inlet and outlet connection ports $(\phi 2.9 \mathrm{~mm})$ were cut for direct tubing (1/16" OD) connections or docking with the connectors on the off-chip interface; (v) sensing manifold comprising of an additional layer with embedded Presens Sensor Foils (Figure 1C-E). The sensor foils were made of a plastic backing coated with a non-consuming, non-binding oxygen fluorescing substance that was indicative of oxygen tension (i.e., partial pressure of oxygen as dissolved gas in an aqueous solution) in a two dimensional plane (Figure 1) ${ }^{4-6}$. The indicator dye was emitting fluorescence that was dynamically quenched by oxygen while the reference dye was emitting a constant green light signal ${ }^{4-6}$. The sensor foils were placed on the bottom plane of the chip-based device and in direct contact with the immobilized embryos (Figure 1C-E).

\subsection{Oxygen detection}

Oxygen detection was accomplished through the use of a Presens ${ }^{\circledR}$ VisiSens ${ }^{\mathrm{TM}}$ system that detects sensor foil signals using a miniaturised USB-based digital microimaging system (Figure 1D) ${ }^{4-6}$. The detector unit consisted of a portable USB microscope (magnification 10X or greater depending on the adaptors being used) connected to a laptop computer (Toshiba Satellite P70) (Figure 1D). The acquisition software (VisiSens Analytical 1, ver. 1.13) supported capture of 1.3 megapixel images at up to 0.5 frames per second (fps) (Figure 1D). The software allowed calculation of oxygen levels on this foil through calibration of fluorescence readings with known oxygen levels as analysed. Specifically, the detector 
unit was designed to measure oxygen tension between 0 and $100 \%$ air saturation (i.e., $20.9 \% \mathrm{O}_{2}$ of all dissolved gas by volume) between 5 and $45^{\circ} \mathrm{C}$. This allowed two-dimensional (2D) visualization of oxygen distribution over the imaging area in real time, to the limits of the imaging resolution. The acquired color-coded oxygen 2D maps permitted a quantitative analysis of distinct tissue regions. In addition to high spatial and temporal resolution, the advantages of this new technology included signal independence of liquid flow velocity. For all experiments, extraneous light that could have interfered with the sensing device was blocked to the chip by the use of black opaque box that fit over the entire microfluidic system and VisiSens ${ }^{\mathrm{TM}}$ microscope/camera. All solutions were thermostatted to $28^{\circ} \mathrm{C}$ using a digitally controlled water bath. The chip-based device was monitored using an infrared temperature sensor to ensure temperature did not change throughout the experimental protocol.

\section{Figure 1}

A)

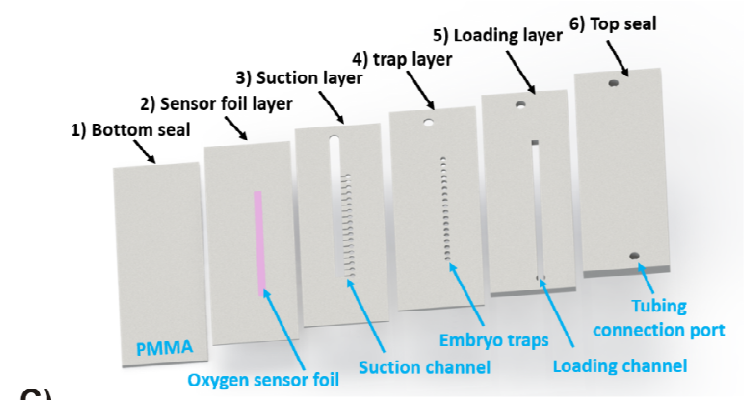

C)
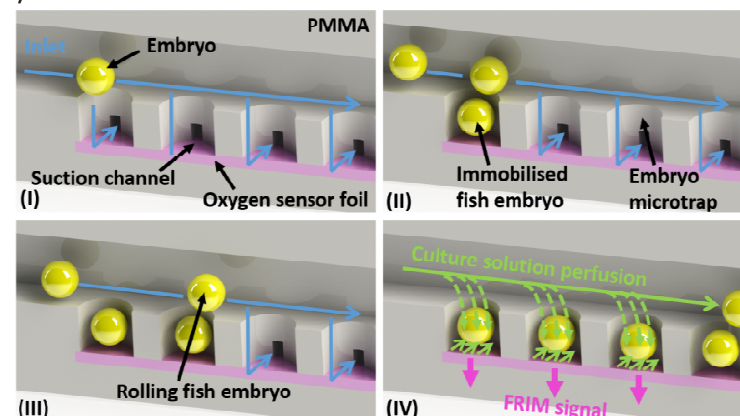

(III)

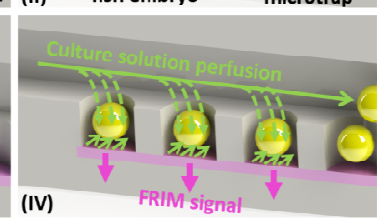

B)

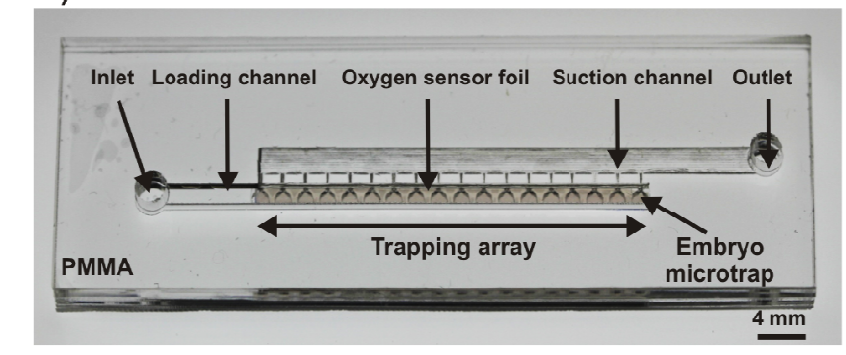

D)

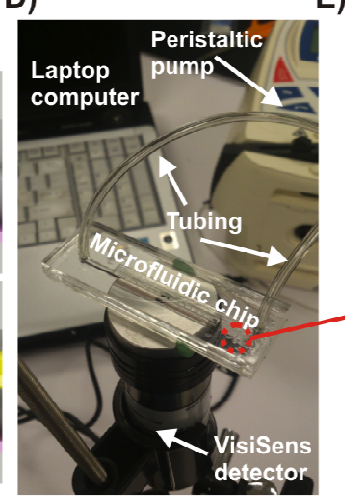

E)

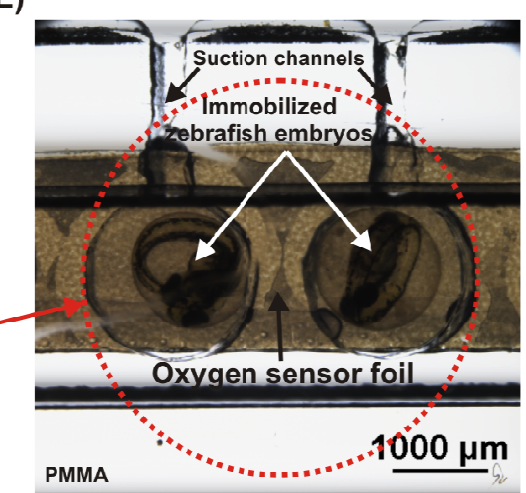

Figure 1. Microfluidic chip-based system. A) An exploded 3D CAD view of the microfluidic chip depicting all layers; B) A photomicrograph of the chip-based device with embryo traps. All features depicted; C) 3D cartoon depicting the process of embryo trapping and docking principles: i) System exploits combined gravitational-induced sedimentation and low pressure suction at the bottom of the embryo trap; ii) and iii) Trapped embryos act as plugs enabling following embryos to roll over and toward next available trap; iv) After all embryos are trapped, microperfusion that can be performed in both closed-loop or open-loop regiments can be initiated; D) A photograph depicting Presens ${ }^{\circledR}$ VisiSens ${ }^{\mathrm{TM}}$ system integrated with the microfluidic chip-based device. The detector unit consisted of a portable USB microscope with 1.3MP CMOS chip and magnification 10X or greater depending on the adaptors; E) Magnified view of a chip-based device loaded with developing zebrafish embryos.

\subsection{Calibration and data analysis}

Prior to loading the embryos, the oxygen sensors required a two-step calibration. Accordingly, the chip was perfused with a solution of air-saturated water (via vigorous bubbling with air, $\mathrm{DO} \approx 9 \mathrm{mg} \mathrm{O}_{2} / 1$ water) and each well was imaged four times. Following this, the chip was perfused with a chemically induced anoxic water $\left(\mathrm{Na}_{2} \mathrm{SO}_{3}: 5 \mathrm{~g} / \mathrm{l}, \mathrm{DO}=0 \mathrm{mg} \mathrm{O} / 1\right.$ water), and the well imaged a further four times. Multiple images were taken to ensure response time had been reached. Large areas of the wells from the appropriate images were selected as a region of interest (ROI) and the resulting fluorescence values were incorporated into the calibration curve calculation provided by the software. The fluorescence at $100 \%$ and $0 \%$ oxygen saturation were used to create a linear regression that described how fluorescence changed with 
$\mathrm{O}_{2}$ tension. To obtain measurements from the oxygen sensing foils, images were taken in sequence and fluorescence within a ROI was recorded over the entire time course of the experiment. Fluorescence values were then entered into a calibration equation within the software. For calibration, ROIs were selected from the entire well, although fluorescence values and calibration curves were similar between experiments. For experiments, ROI were selected from the region proximal to the embryo.

\section{RESULTS AND DISCUSSION}

To investigate how altering supply of oxygen would affect oxygen gradients under resting metabolic demand, embryos (between 30 and $40 \mathrm{hpf}$ ) were loaded onto the chips, protected from ambient light, and allowed to acclimatize to their new environment for 30 minutes at a high rate of solution perfusion. After this period, perfusion rates were set to a specific flow rate, a short period was given to allow the new flow rate to become stable, and measurements were then taken for two minutes, each measurement being 10 seconds apart. This protocol was performed with different embryos $\left(\mathrm{n}=10\right.$ each time) at three different flow rates (approximately $0.4,0.1$, and $\left.0.05 \mathrm{ml} \mathrm{min}^{-1}\right)$, randomly ordered. These flow rates were selected based on preliminary work demonstrating that oxygen gradients could be increased when flow rates were reduced to these levels.

To characterise how oxygen gradients could be perceived during depletion of oxygen and recovery of pre-depletion gradients, embryos were observed during cessation and reinstatement of flow of oxygen saturated water. After embryos were loaded in the chips, 30 minutes was allowed to pass, and then all flow to the embryos was shut off for $120 \mathrm{sec}$. Perfusion was then restarted at $0.4 \mathrm{ml} / \mathrm{min}$. Measurements were obtained in a similar manner as that described above (24 measurements taken once every 10 seconds).

Oxygen sensitivity as observed during calibration was high, with substantial changes occurring rapidly with the introduction of the anoxia solution (Figure 2A). However, the excessive fluorescence of the oxygen sensing foils when exposed to air confirmed that calibration in air was inappropriate due to the much greater oxygen content of air compared to water (Figure 2B), and emphasized the necessity of ensuring that solutions were free of air bubbles. Over the course of the experiments, all embryos survived experimental treatments. No phenotypic i.e. morphological abnormalities were noticeable during embryo development on the chip. The cumulative survival of embryos that were immobilized on a chip-based device was over $99 \pm 1 \%$. In comparison, in control experiments performed on a Petri dish the cumulative survival of embryos was also over $99 \pm 1 \%$. Moreover, no discernible phenotypic effects on cardiovascular function (e.g. heart rate) and behavioural traits were observed in recovered specimens that were immobilized on a chipbased device and all parameters were statistically comparable with control groups kept in static Petri dish control experiments. This was despite observing $\mathrm{O}_{2}$ tensions proximal to the embryo as low as $5 \%$ of air saturation for periods of several minutes. Oxygen levels in wells without embryos did not drop over the course of the experiment.

By altering flow, we were able to alter the concentration of oxygen in the proximal contact zones of the zebrafish embryos. Proximal ROIs were determined through directly assessing embryo positioning from light imagery, and outlining that entire area of the chorion in the fluorescent image (see representative image collage, Figure 2C). At the highest flow rates, oxygen levels appeared to be relatively stable over the $120 \mathrm{sec}$ trial. In contrast, when we reduced flow by $1 / 2$ or $3 / 4$ (from 0.4 to 0.2 or $0.1 \mathrm{ml} / \mathrm{min}$ ) oxygen levels dropped from approximately $100 \%$ after $120 \mathrm{sec}$ to $50 \%$ or $20 \%$ respectively (Figure 2D,E), thus indicating a mismatch between $\mathrm{O}_{2}$ demand and supply. When flow $(0.4 \mathrm{ml} / \mathrm{min})$ to the embryo was ceased, oxygen levels fell rapidly in the areas close to the embryos, such that over 120 seconds, anoxic zones (oxygen levels of less than 5\% air saturation) were created proximal to the embryos (Figure 2F). When water flow was reintroduced, oxygen levels surrounding the embryo slowly increased for the first $30 \mathrm{sec}$, but then increased dramatically and had returned to normal within $60 \mathrm{sec}$ of reintroducing flow at the initial rate $(0.4 \mathrm{ml} / \mathrm{min}$, Figure $2 \mathrm{~F})$.

Oxygen sensing using embedded on-a-chip sensor foil technology was deemed sufficient to record clear oxygen gradients surrounding zebrafish embryos. Thus it is feasible that, if estimates of oxygen content available to the embryo can be made based on chamber size and flow rates of water, then using predictive diffusion models, $\mathrm{MO}_{2}$ of individual embryos will also be able to be estimated from these images ${ }^{11}$. Certainly relative oxygen consumption rates may be estimated from changes in $\mathrm{O}_{2}$ levels or gradients surrounding the embryos, and when used in conjunction with other recent experimental protocols, such as multiwell assays; ${ }^{12}$ could provide remarkable insight into oxygen uptake dynamics. Of particular interest might be, for example, how changes in laminar flow, particular concentrations (i.e., sediment levels), temperatures or water $\mathrm{pH}$ affect oxygen gradients. In our hands, integration of sensor foil oxygen 
detection system (VisiSens ${ }^{\mathrm{TM}}$, PreSens ${ }^{\circledR}$, Germany) with a microfluidic chip-based embryo array was highly responsive and, although it may currently lack the resolution needed for 2D mapping capacity, we believe it can ultimately be effective as a tool for investigation into metabolic studies focused on stationary aquatic organisms similar in size and/or oxygen consumption rates to the zebrafish embryos examined here. Undoubtedly future work is needed especially focused on applications of much higher resolution and more sensitive CMOS sensors used in the detector unit.

\section{Figure 2}

A)

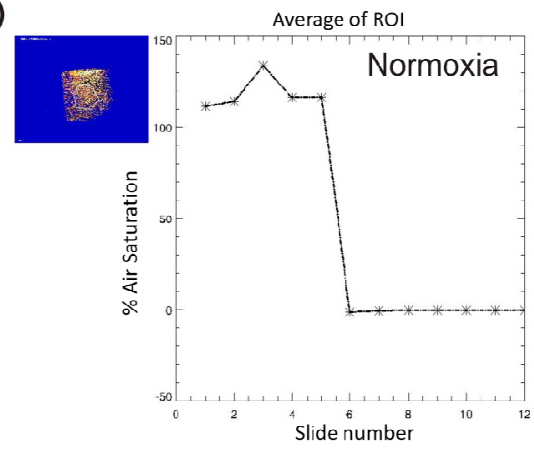

D)

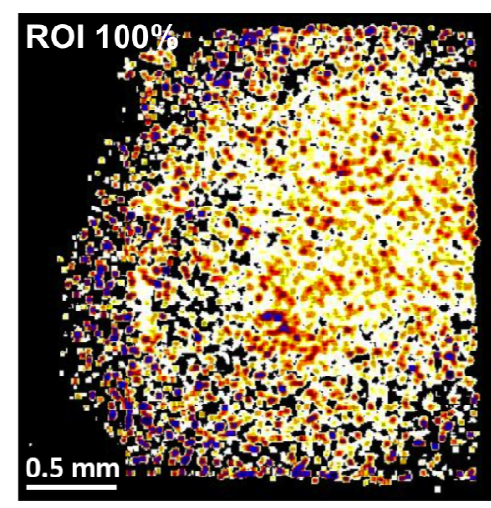

E)

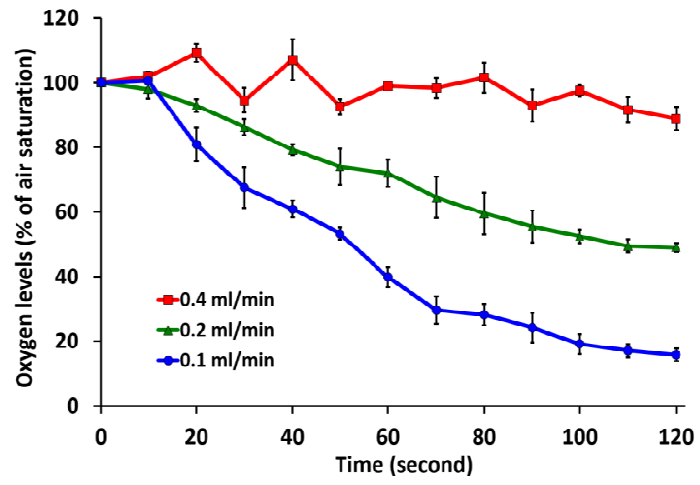

B)

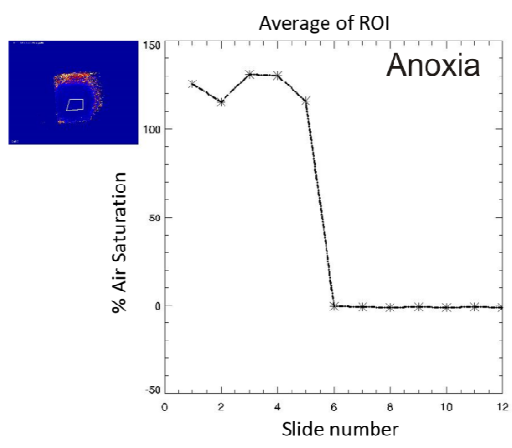

C)

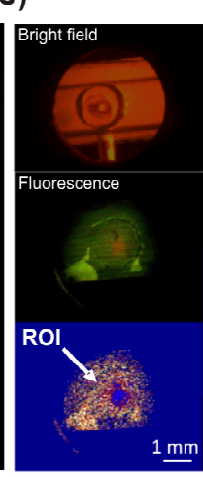

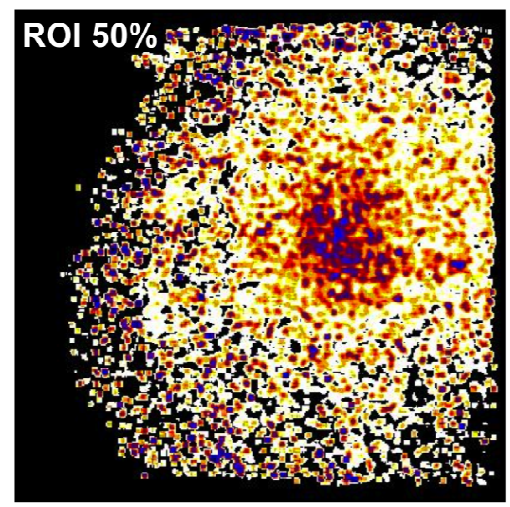

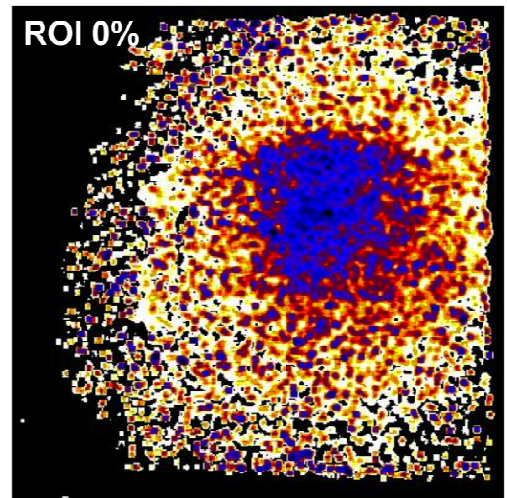

F)

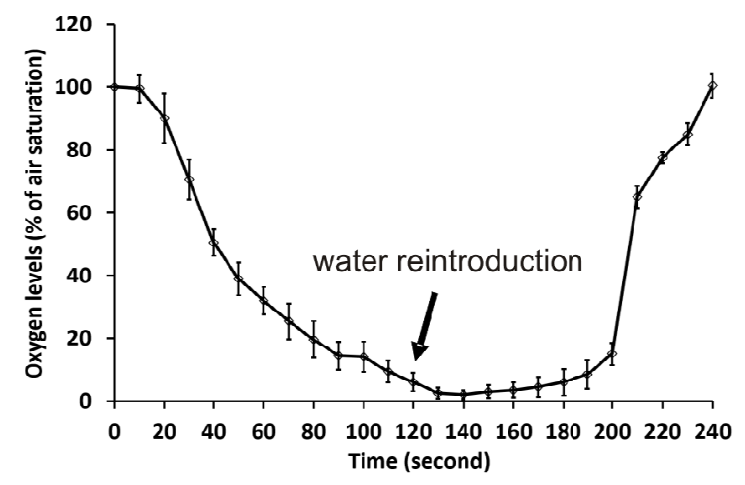

Figure 2. Detection of oxygen gradients on a chip-based device using embedded sensor foils. A) Oxygen saturation as a percentage of ambient air oxygen levels during exposure to normoxic and anoxic solutions. The image on the left is fluorescence during normoxia, while that on the right is during anoxia exposure. Slide number represents each measurement at 10 -second intervals. Regions of interest (ROI) were taken from areas not used for calibration. Note the rapid change in fluorescence from normoxia to anoxia. Note also the homogeneity of the signal when no oxygen is present compared to normoxic levels, a characteristic of detection of fluorescent signaling; B) Representative diagrams of a light image (top panel) and a fluorescence image (bottom panel) obtained from the VisiSens camera of a drop of anoxic water on the oxygen sensing foils. In the top image the boundary between anoxic (orange) and normoxic (green) can be clearly seen. However, the subtleties of this image are much more clearly observed in the fluorescence image below that. Note the extremely high 
fluorescence on the left of the bottom image, indicative of the high rates of diffusion in air relative to water. This excessive fluorescence demonstrates that calibration in air is not possible; C) Representative image (light on top, fluorescence in middle, calculated oxygen levels at bottom) of zebrafish embryo with oxygen gradients due to reduced water flow; D) Representative images of calculated oxygen levels (\% air saturation) in $40 \mathrm{hpf}$ zebrafish embryos consuming oxygen during cessation of flow. From left to right: ROI 100\% (0 seconds after cessation of microperfusion); ROI $50 \%$ (40 sec after cessation of microperfusion) and ROI $0 \%$ (140 sec after cessation of microperfusion); E) The effect of flow (0.4, 0.2 and 0.1 $\mathrm{ml} / \mathrm{min}$ ) on oxygen levels over time in proximal (contact) zones of $40 \mathrm{hpf}$ zebrafish embryos. $\mathrm{n}=3$ or 4 for each line. Error bars represent standard errors; F) Oxygen (\% air saturation) as a function of time (seconds) during the absence and reintroduction of water flow to $40 \mathrm{hpf}$ zebrafish embryos in microfluidic chips. Error bars represent standard errors. $\mathrm{n}=3$ for each point.

\section{CONCLUSIONS}

In this work, we demonstrated a proof-of-concept approach for non-invasive and real-time visualisation of metabolic activities by using microfluidic Lab-on-a-Chip (LOC) technologies combined with optoelectronic sensors. It was achieved by immobilising live zebrafish embryos with continuous flow perfusion, while using Fluorescence Ratiometric Imaging (FRIM) technology to quantify the temporal patterns of aqueous oxygen gradients kinetically from an optical sensor. We postulate that this technology will be an effective tool for metabolic studies focused on stationary aquatic organisms similar in size and/or oxygen consumption rates to the zebrafish embryos examined here. Future work is needed on applications of much higher resolution and more sensitive CMOS sensors used in the detector unit.

\section{REFERENCES}

[1] Santoro, R., Krause, C., Martin, I., and Wendt, D., "On-line monitoring of oxygen as a non-destructive method to quantify cells in engineered 3D tissue constructs," Journal of tissue engineering and regenerative medicine, (2011).

[2] Tengberg, A., Hovdenes, J., Andersson, J. H., Brocandel, O., Diaz, R., Hebert, D., Arnerich, T., Huber, C., Körtzinger, A., Khripounoff, A., Rey, F., Rönning, C., Schimanski, J., Sommer, S., and Stangelmayer, A., "Evaluation of a lifetime-based optode to measure oxygen in aquatic systems.," Limnology and Oceanography: Methods(4), 7-17 (2006).

[3] Okubo, N., Yamamoto, H. H., Nakaya, F., and Okaji, K., "Oxygen consumption of a single embryo/planula in the reef-building coral Acropora intermedia.," Marine Ecology Progress Series(366), 305-309 (2008).

[4] Meier, J. K., Gosau, M., Müller, S., Moralis, A., Liebsch, G., and Prantl, L., [Perfusion Monitoring in Microvascular Flaps: Fluorescence Imaging Applied as a Method to Discover Vessel Thrombosis.], (2012).

[5] Meier, J. K., Prantl, L., Muller, S., Moralis, A., Liebsch, G., and Gosau, M., "Simple, fast and reliable perfusion monitoring of microvascular flaps," Clinical hemorheology and microcirculation, 50(1-2), 13-24 (2012).

[6] Kellner, K., Liebsch, G., Klimant, I., Wolfbeis, O. S., Blunk, T., Schulz, M. B., and Gopferich, A., "Determination of oxygen gradients in engineered tissue using a fluorescent sensor," Biotechnology and bioengineering, 80(1), 73-83 (2002).

[7] Zhu, F., Skommer, J., Huang, Y., Akagi, J., Adams, D., Levin, M., Hall, C. J., Crosier, P. S., and Wlodkowic, D., "Fishing on chips: Up-and-coming technological advances in analysis of zebrafish and Xenopus embryos," Cytometry. Part A : the journal of the International Society for Analytical Cytology, (2014).

[8] Akagi, J., Zhu, F., Hall, C. J., Crosier, K. E., Crosier, P. S., and Wlodkowic, D., "Integrated chip-based physiometer for automated fish embryo toxicity biotests in pharmaceutical screening and ecotoxicology," Cytometry. Part A : the journal of the International Society for Analytical Cytology, 85(6), 537-47 (2014).

[9] Wlodkowic, D., Khoshmanesh, K., Akagi, J., Williams, D. E., and Cooper, J. M., "Wormometry-on-a-chip: Innovative technologies for in situ analysis of small multicellular organisms," Cytometry. Part A : the journal of the International Society for Analytical Cytology, 79(10), 799-813 (2011).

[10] Akagi, J., Khoshmanesh, K., Hall, C. J., Cooper, J. M., Crosier, K. E., Crosier, P. S., and Wlodkowic, D., "Fish on chips: Microfluidic living embryo array for accelerated in vivo angiogenesis assays," Sensors and Actuators B-Chemical, 189, 11-20 (2013).

[11] Lighton, J. R. B., [Measuring metabolic rates: a manual for scientists.] Oxford University Press, (2008).

[12] Stackley, K. D., Beeson, C. C., Rahn, J. J., and Chan, S. S., "Bioenergetic profiling of zebrafish embryonic development," PLoS One, 6(9), e25652 (2011). 